\title{
Thermal Illusion of Porous Media with Convection-Diffusion Process: Transparency, Concentrating, and Cloaking
}

\author{
Fubao Yang, Liujun $\mathrm{Xu}^{*}$ and Jiping Huang*
}

Thermal convection-diffusion plays a crucial role in transferring heat energy in the nature, and hence its effective manipulation is of great significance. Although the transformation theory provides a possible approach, three severe problems still restrict practical applications. That is, the parameters as required by the transformation theory are anisotropic, inhomogeneous, and even singular, thus challenging the fabrications. To solve these problems, here we propose a scheme for realizing thermal illusion (including transparency, concentrating, and cloaking) of porous media with convection-diffusion process, which is governed by Darcy's law and Fourier's law. By designing two key parameters (i.e., thermal conductivity and permeability) of a shell, we can realize thermal transparency and remove the requirements of anisotropy, inhomogeneity, and singularity. The scheme can also help to realize thermal concentrating or cloaking by designing an anisotropic shell without the need of inhomogeneous and singular parameters. All these theoretical analyses are confirmed by finite-element simulations. This work provides an illusion scheme for manipulating thermal convection-diffusion with practical parameters, and offers a guidance to fabricate these metamaterials experimentally. Our results can also be extended to other diffusive fields, such as mass diffusion.

Keywords: Thermal illusion; Thermal convection-diffusion; Porous media; Microfluidics

Received 22 August 2019, Accepted 29 September 2019

DOI: $10.30919 /$ esee8c329

\section{Introduction}

Heat flow exists almost everywhere in the nature, and hence its effective management is of particular significance. Fortunately, the past years have witnessed the development of manipulating heat conduction at the nanoscale. Many computational methods have been developed, such as first-principles Boltzmann transport equation, molecular dynamics, non-equilibrium Green's function, and numerical solution of phonon Boltzmann transport equation. ${ }^{1}$ These methods provide a promising approach to manipulating heat flow.

Meanwhile, researchers are also devoted to understanding and controlling heat flow at the macroscale. In 2008, Fan et al. ${ }^{2}$ proposed thermal cloaking based on the approach of coordinate transformation. Heat flow can be guided to keep off an "invisible" region at steady states without distorting the temperature distribution of the background. From then on, many thermal metamaterials have been theoretically designed and experimentally realized in heat conduction, such as extensional thermal cloaking, ${ }^{3-9}$ thermal concentrating, ${ }^{10-14}$ thermal transparency, ${ }^{15-17}$ thermal camouflage/illusion, ${ }^{18-25}$ and even thermally responsive metamaterials. ${ }^{26}$ When considering the coupling between thermal convection and diffusion (conduction), the transformation approach also works. Some recent work ${ }^{27,28}$ established the transformation theory for thermal convection-diffusion process in

Department of Physics, State Key Laboratory of Surface Physics, and Key Laboratory of Micro and Nano Photonic Structures (MOE), Fudan University, Shanghai 200438, China

*E-mail: 13307110076@fudan.edu.cn; jphuang@fudan.edu.cn porous media. Thus, the theoretical treatment based on the coordinate transformation (called transformation thermotics) has become a useful method for manipulating macroscopic heat flow at will.

However, most metamaterials designed by transformation thermotics have inevitably anisotropic, inhomogeneous, and even singular parameters, thus challenging the fabrications. To solve these problems in thermal diffusion, people use effective medium theories and multilayered composite structures to realize the desired functions. ${ }^{4,5}, 29$ Unfortunately, there is not an appropriate theory for handling thermal convection-diffusion so far. Thus, it becomes urgent to establish a theory to simplify the complex parameters given by transformation thermotics.

For this purpose, we refer to the concept of neutral inclusion, ${ }^{15}$ and extend it to thermal convection-diffusion of microfluidics in porous media. We consider two physical quantities (i.e., thermal conductivity and permeability) and calculate their respective effective values. By designing these two key parameters, we realize three types of thermal illusion (including transparency, concentrating, and cloaking). Concretely speaking, thermal transparency is to construct a core-shell structure in order not to disturb the temperature, velocity, and heat flux distributions in the background. Especially, it can remove the requirements of anisotropy, inhomogeneity, and singularity. On the same footing, for achieving thermal concentrating or cloaking, we design an anisotropic shell with no need to adopt inhomogeneous and singular parameters. Since transparency, concentrating, and cloaking do not disturb the temperature, velocity, and heat flux distributions in the background, we collectively call them thermal illusion for convenience in this work. Finally, we perform finite-element simulations with software COMSOL $5.4^{30}$ to confirm our theory. 


\section{Theoretical methods}

We consider a passive thermal convection-diffusion process in porous media with an incompressible fluid and neglect the viscous dissipation term. Then, the thermal convection-diffusion equation can be expressed $\operatorname{as}^{27}$

$$
\rho_{f} C_{p, f}(\vec{v} \cdot \nabla T)=\nabla \cdot(\stackrel{\leftrightarrow}{\kappa} \cdot \nabla T)
$$

where $\rho_{f}, C_{p, f}$, and $\vec{v}$ are, respectively, the density, heat capacity, and velocity of the fluid at constant pressure, and $T$ represents the temperature when the porous media and the fluid reach equilibrium. In addition, $\overleftrightarrow{\kappa}$, which denotes the average thermal conductivity tensor of the porous media and the fluid, is given by $\overleftrightarrow{\kappa}=(1-\phi) \overleftrightarrow{\kappa}_{s}+\phi \overleftrightarrow{\kappa}_{f}$, where $\phi$ is the porosity of the media. $\overleftrightarrow{\kappa}_{s}$ and $\overleftrightarrow{\kappa}_{f}$ are the thermal conductivity tensors of the solid porous media and fluid, respectively. The left part of Eq. (1) is the term of thermal convection. Although Eq. (1) seems that the permeability and thermal conductivity are coupled together, we have to claim that they are intrinsic properties of materials. In other words, Eq. (1) just describes the thermodynamic state of fluids after we preset the thermal conductivity and permeability. Thus, we can consider thermal diffusion and thermal convection independently. If the fluid is laminar with very slow speed, the velocity $\vec{v}$ is determined by Darcy's law,

$$
\vec{v}=-(\stackrel{\leftrightarrow}{\sigma} / \eta) \cdot \nabla p
$$

where $\stackrel{\leftrightarrow}{\sigma}$ and $\eta$ denote the permeability tensor and dynamic viscosity, respectively. $p$ denotes pressure. Eq. (2) is valid under the condition that both Re (Reynolds number) and $\vec{J}$ are low enough. The conductive flux is given by Fourier's law,

$$
\vec{J}=-\stackrel{\leftrightarrow}{\kappa} \cdot \nabla T
$$

For simplicity, we consider the passive states, thus yielding

$$
\begin{aligned}
& \nabla \cdot \vec{v}=0, \\
& \nabla \cdot \vec{J}=0 .
\end{aligned}
$$

We also consider one type of fluid which means that dynamic viscosity $\eta$ is constant. Then, Eqs. (4) and (5) can be expressed as

$$
\begin{aligned}
& \nabla \cdot(-\stackrel{\leftrightarrow}{\sigma} \cdot \nabla p)=0 \\
& \nabla \cdot(-\stackrel{\leftrightarrow}{\kappa} \cdot \nabla T)=0
\end{aligned}
$$

Clearly, Eqs. (6) and (7) have the same mathematical form. Thus, the effective medium theory can handle both thermal conductivity and permeability. We can use $\tau$ to unify the representation of $\kappa$ and $\sigma$.

Our aim is to remove the requirements of anisotropic, inhomogeneous, and singular parameters. Therefore, we refer to the concept of neutral inclusion. ${ }^{15}$ This concept gives us a way to calculate the effective thermal conductivity of a core-shell structure. Then, we need to calculate the effective permeability of the same core-shell structure. As shown in Fig. 1, we set the core to be isotropic with parameter $\tau_{1}$, the shell to be anisotropic with parameter $\stackrel{\leftrightarrow}{\tau_{2}}=\operatorname{diag}\left(\tau_{r r}=\tau_{\theta \theta}\right)$ (the shell becomes isotropic when $\tau_{r r}=\tau_{\theta \theta}$ ), and the background to be isotropic with parameter $\tau_{3}$. Then, the effective parameter $\tau_{e}$ of the coreshell structure can be calculated as

$$
\tau_{e}=C \tau_{r r} \frac{\tau_{1}+C \tau_{r r}+\left(\tau_{1}-C \tau_{r r}\right) f^{c}}{\tau_{1}+C \tau_{r r}-\left(\tau_{1}-C \tau_{r r}\right) f^{c}},
$$

where $C=\sqrt{\tau_{\theta \theta} / \tau_{r r}}$ denotes the anisotropy of the shell, and $f=\left(r_{1} / r_{2}\right)^{2}$ denotes the core fraction. We set $\tau_{e}=\tau_{3}$ to ensure that the heat flux and velocity distributions in the background (region III) behave as if there were not a core-shell structure in the center.

\section{Results}

In this section, we present finite-element simulations to validate our theoretical calculations. Because of the same equation form of $\kappa$ and $\sigma$, we can easily conclude that the effective permeability should have the same mathematical expression as the effective thermal conductivity. We theoretically derive the conditions of thermal convection-diffusion transparency in two dimensions, say, $\tau_{e}\left(\kappa_{e}\right.$ or $\left.\sigma_{e}\right)=\tau_{3}\left(\kappa_{3}\right.$ or $\left.\sigma_{3}\right)$. Thermal transparency requires that the temperature, velocity, and heat flux distributions in region III exhibit the same profile as if there was not a core-shell structure in the center. We validate the above theory by using the templates of heat transfer in porous media and Darcy's law in COMSOL. Without loss of generality, we take the pressure field parallel or perpendicular to the temperature field; see Fig. 1.
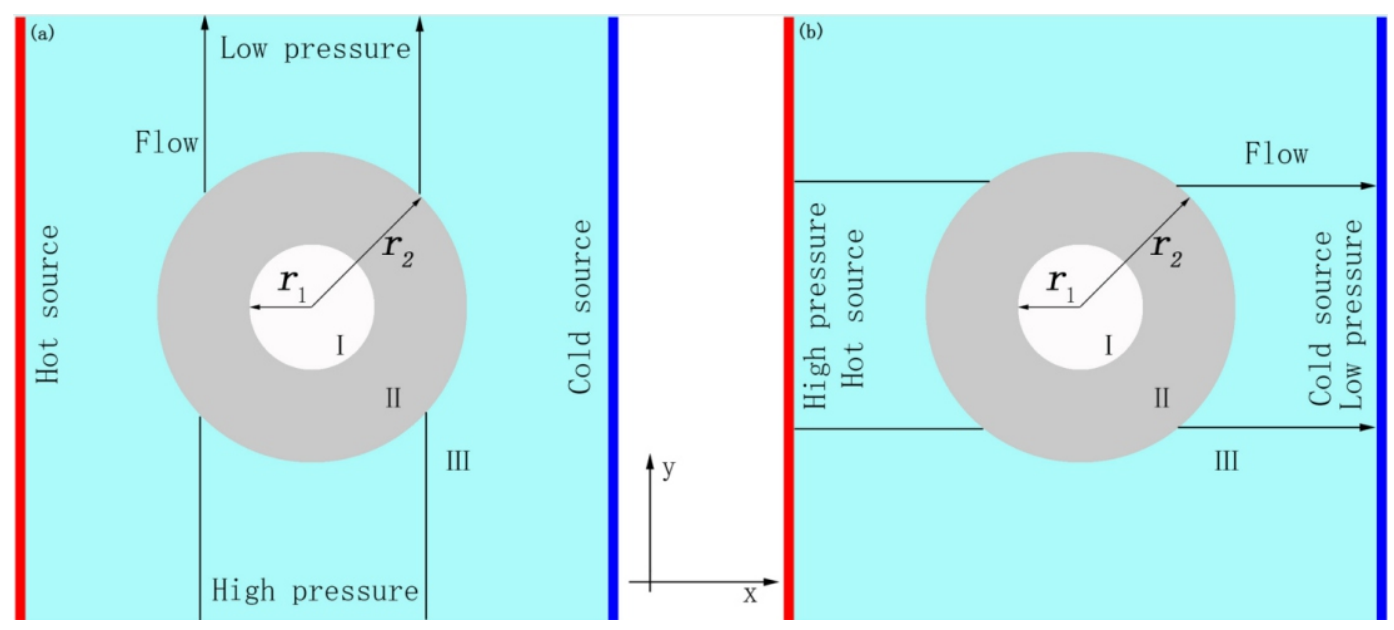

Fig. 1 Schematic diagrams of thermal illusion. The scale of the system is $10^{-5} \mathrm{~m}$. (a) The background velocity is along the $y$ direction, as described by the black flow lines. (b) The background velocity is along the $x$ direction. Region I $\left(r<r_{1}\right)$ is composed of isotropic porous media, region II $\left(r_{1}<r<r_{2}\right)$ is composed of isotropic media for thermal transparency, and anisotropic media for thermal concentrating or cloaking, and region III $\left(r>r_{2}\right)$ is composed of isotropic background porous media. For thermal illusion in porous media, the black lines in region III with the core-shell structure should be undistorted. 
In all simulations, we take $\Delta p=400 \mathrm{~Pa}$ and $\Delta T=40 \mathrm{~K}$. The fluid in porous media is set as water with $\rho_{f}=10^{3} \mathrm{~kg} / \mathrm{m}^{3}, C_{p, f}=4.2 \times 10^{3} \mathrm{~J} \mathrm{~kg}^{-1} \mathrm{~K}^{-1}$, the dynamic viscosity $\eta=10^{-3} \mathrm{~Pa} \cdot \mathrm{s}$, and $\kappa_{f}=0.6 \mathrm{Wm}^{-1} \mathrm{~K}^{-1}$. The porosity of the whole region is $\phi=0.9$. The size parameters are $r_{1}=10^{-5} \mathrm{~m}$ and $r_{2}=10^{-5} \mathrm{~m}$. The average thermal conductivity tensors are set to be $\kappa_{1}=6$ $\mathrm{Wm}^{-1} \mathrm{~K}^{-1}, \stackrel{\leftrightarrow}{\kappa_{2}}=\operatorname{diag}(4,4) \mathrm{Wm}^{-1} \mathrm{~K}^{-1}$, and $\kappa_{3}=\kappa_{e}$ given by Eq. (8). The thermal conductivity tensor of the porous media can be calculated as $\overleftrightarrow{\kappa_{s}}=\overleftrightarrow{\leftrightarrow}$ $\left.\phi \kappa_{f}\right) /(1-\phi)$ The permeability tensors are set to be $\sigma_{1}=5 \times 10^{-12} \mathrm{~m}^{2}, \stackrel{\leftrightarrow}{\sigma_{2}}=\operatorname{diag}(2$, 2) $\times 10^{-12} \mathrm{~m}^{2}$ (the magnitude $10^{-12}$ is common in nature), and $\sigma_{3}=\sigma_{e}$ by Eq. (8). In all these cases, we set Reynolds numbers $\operatorname{Re}=r_{2} \rho_{f} v / \eta<1$ (the maximum value is 0.64 ) and $\sigma_{2} \ll r_{2}^{2}$, so Darcy's law is valid.

The simulation results are shown in Figs. 2 and 3. The pressure field in Fig. 2 is along the $y$ direction, and the second row is a reference without the core-shell structure. Comparing the first and the second rows, we find the distributions of temperature, velocity, and heat flux are the same in region III, as if there were not a device in the background. These results validate the above theory of thermal transparency. The conclusion is the same when the pressure field is parallel to the temperature field. Besides, Figs. 2(c) and 2(f) show that the total heat flux is curved. This is because the direction of convection flux is perpendicular to that of the conduction flux. The vertical velocity field introduced by thermal convection just gives the total heat flux a vertical component. It has no effect on the horizontal heat flux field and temperature field, so it does not affect the temperature distribution, but affects the distribution of heat flux. What's more, the changes of temperature difference and pressure difference only cause different heat flow field and velocity field. The effects of thermal illusion will not be affected as long as the permeability and thermal conductivity satisfy Eq. (8).

Since we take the permeability at the order of magnitude $10^{-12}$, the heat flux caused by convection may be too small to have an effect on the distributions of temperature, velocity, and heat flux. We only change the permeability of region III in Fig. 2. If the permeability of the background is not equal to the value calculated by Eq. (8), the function of thermal transparency will disappear; see Fig. 4. Thus, the results of Fig. 4 also validate our theory. Then, we can conclude that only when the parameters (thermal conductivity and permeability) of the three regions satisfy Eq. (8), can the thermal transparency appear for creeping flow in porous media. Therefore, we realize thermal convectiondiffusion transparency with isotropic, homogeneous and nonsingular materials.

In addition, we can also realize thermal concentrating or cloaking by considering a shell with constant anisotropic parameters. $\tau_{r r}$ and $\tau_{\theta \theta}$ represent the ability of heat flow to propagate radially and tangentially, respectively. Thermal concentrating for thermal convection-diffusion can be used to enhance the temperature gradient of a target area. Therefore, we set $\tau_{r r}>\tau_{\theta \theta}$ to realize the concentrating effect. The simulation results are shown in Fig. 5, and heat flow does concentrate at the center region.

With the same thoughts, if we set $\tau_{r r} \ll \tau_{\theta \theta}$, we can realize an
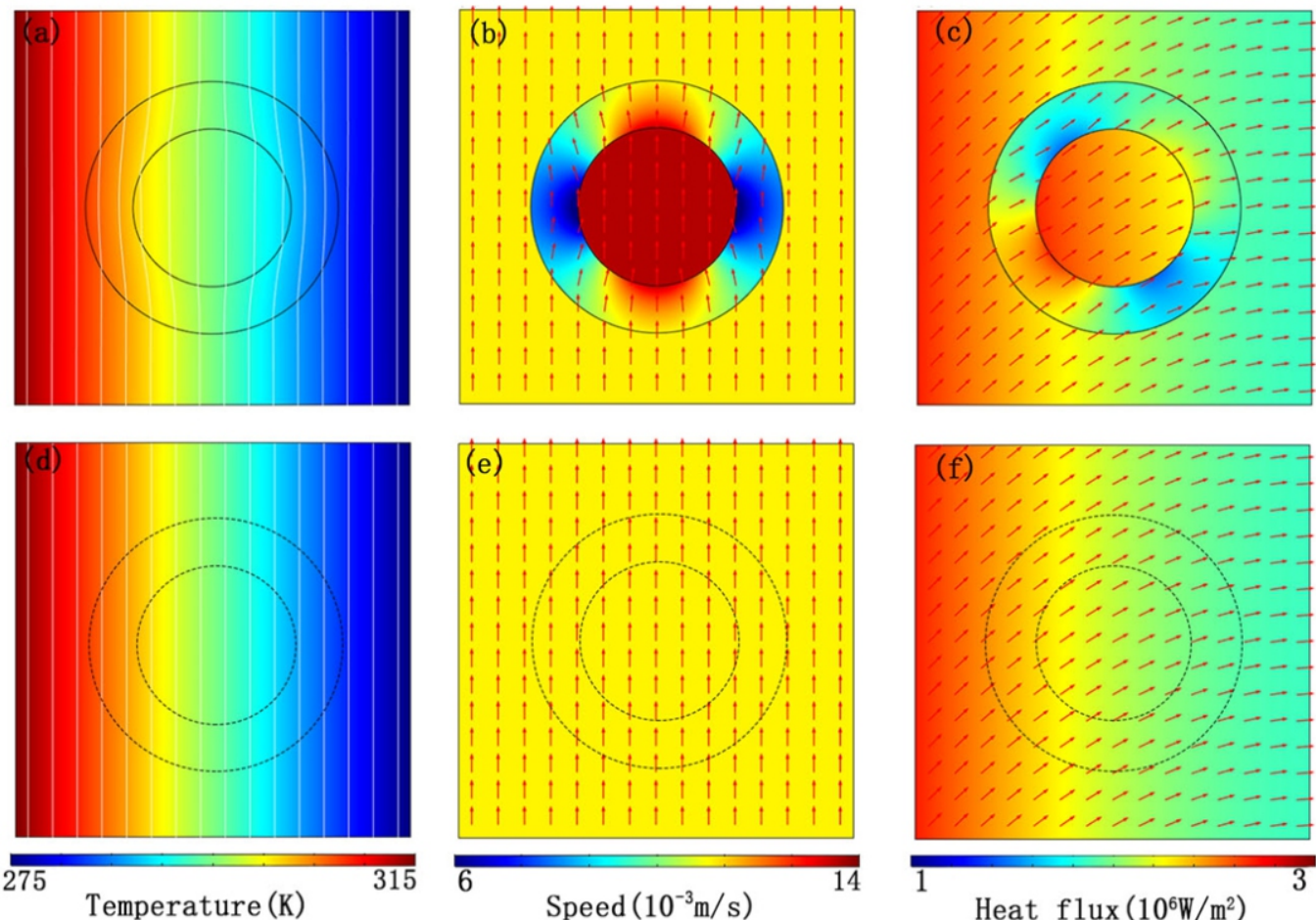

Fig. 2 Simulation results of thermal transparency when the velocity is along the $y$ direction. (a) is the temperature distribution where the white lines are isotherms. (b) shows the distribution of Darcy's velocity whose direction is shown by the arrow direction. (c) describes the distribution of the total heat flux whose direction is shown by the arrow direction. Since the convection flux is vertical to the conduction flux, the total heat flux is curved. Different color maps represent different values, which is shown below each figure. (d)-(f) are the results of pure background for comparison with (a)-(c). 

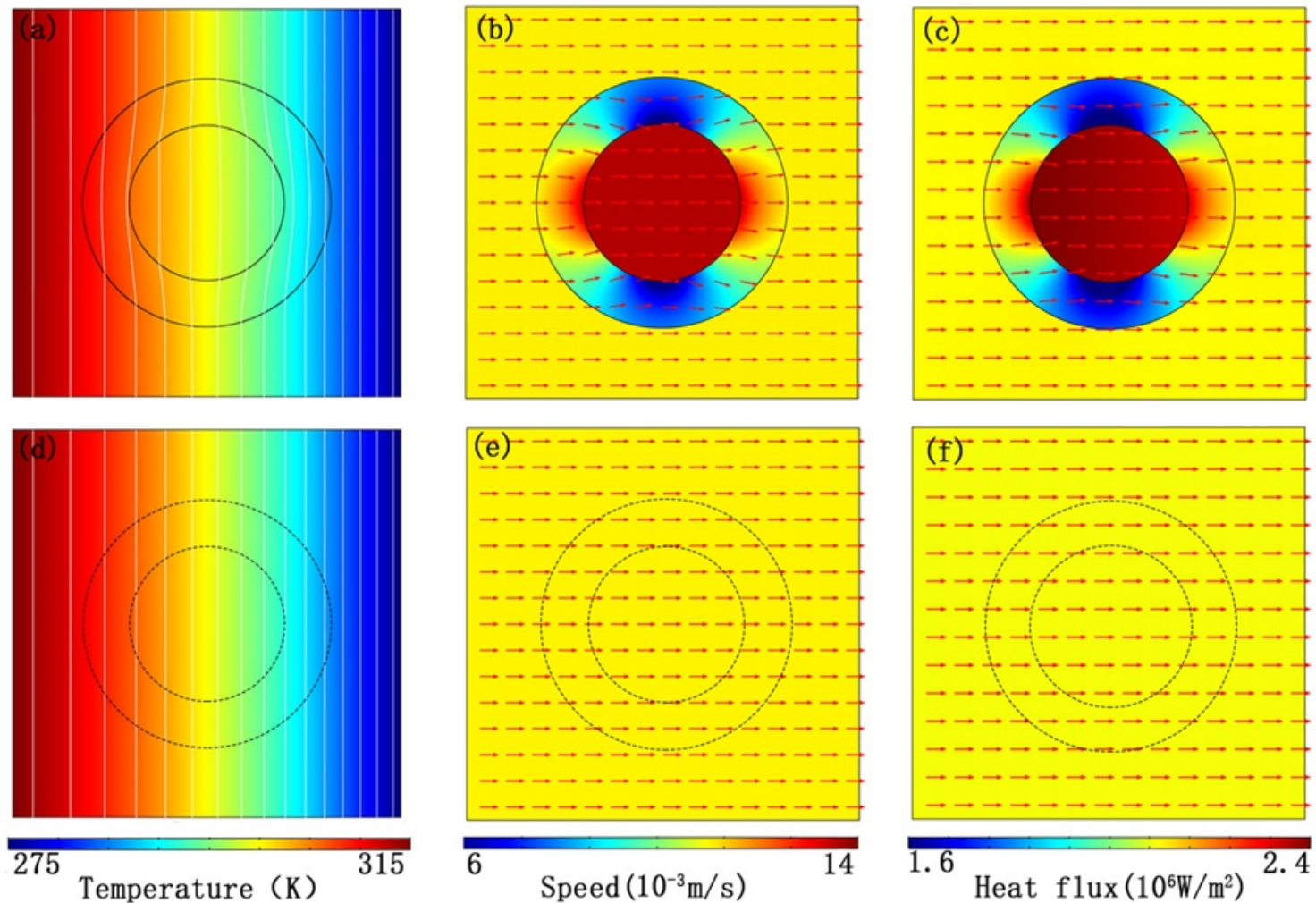

Fig. 3 Simulation results of thermal transparency when the velocity is along the $x$ direction.Other parameters are the same as those in Fig. 2 .
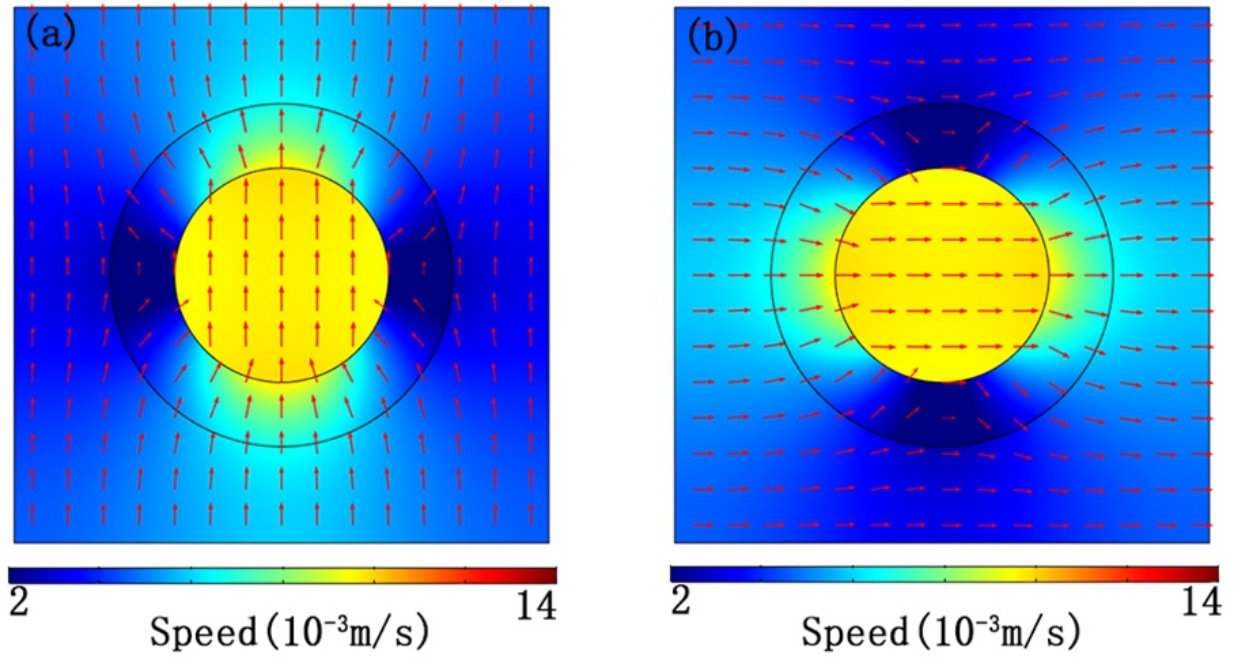

Fig. 4 Simulation results when the permeabilities of the background and core-shell structure mismatch. (a) The distribution of velocity along the $y$ direction in region III is nonuniform, indicating that the core-shell structure is visible when detecting region III and the function of thermal transparency disappears. (b) is the same as (a) except for that the background velocity is along the $x$ direction. 



Fig. 5 Simulation results of thermal concentrating. The background velocity of the first and the second rows are along the $y$ and $x$ directions, respectively. (a)-(c)[or (d)-(f)], respectively, describe the distributions of temperature, velocity and heat flux. The material and size parameters of the background are the same as those in Fig. 2. $\kappa_{1}=1 \mathrm{Wm}^{-1} \mathrm{~K}^{-1}, \overleftrightarrow{\kappa_{2}}=\operatorname{diag}(40,0.4) \mathrm{Wm}^{-1} \mathrm{~K}^{-1}$, and $\kappa_{3}=\kappa_{\mathrm{e}}$ given by Eq. $(8) . \sigma_{1}=10^{-12} \mathrm{~m}^{2}, \stackrel{\leftrightarrow}{\sigma_{2}}=\operatorname{diag}(10,0.1) \times 10^{-12} \mathrm{~m}^{2}$ and $\sigma_{3}=\sigma_{e}$ given by Eq. (8).


Fig. 6 Simulation results of thermal cloaking. The background velocity of the first and the second rows are along the $y$ and $x$ directions, respectively. (a)(c)[or (d)-(f)], respectively, describe the distributions of temperature, velocity and heat flux. The material and size parameters of the background are the same as those in Fig. 2. $\kappa_{1}=1 \mathrm{Wm}^{-1} \mathrm{~K}^{-1}, \overleftrightarrow{\kappa_{2}}=\operatorname{diag}(0.4,40) \mathrm{Wm}^{-1} \mathrm{~K}^{-1}$, and $\kappa_{3}=\kappa_{\mathrm{e}}$ given by Eq. $(8) . \sigma_{1}=10^{-12} \mathrm{~m}^{2}, \stackrel{\leftrightarrow}{\sigma_{2}}=\operatorname{diag}(0.1,10) \times 10^{-12} \mathrm{~m}^{2}$ and $\sigma_{3}=\sigma_{e}$ given by Eq. (8). 
appropriate thermal cloak. When $\tau_{r r}$ exactly reaches zero, an exact cloak can be realized. Although an infinitesimal value of $\tau_{r r}$ still makes the heat flow enter into the target area (region I), it is small enough which can be neglected. Thus, the cloaking effect is also valid. The simulation results are shown in Fig. 6. We find that almost no heat flows into the central region.

According to the effective medium theory, as long as the permeability and thermal conductivity satisfy Eq. (8), the thermal illusion effect will come to appear. In fact, it's not exactly the case. In region II, the anisotropy ratio of the thermal conductivity and permeability should be the same for the thermal transparency effect to occur strictly. It means that $\kappa_{r r} / \kappa_{\theta \theta}=\sigma_{r} / \sigma_{\theta \theta}$. This requirement is in accordance with the transformation theory ${ }^{27}$ because the designed thermal conductivity and permeability should realize the same degree of effect.

Moreover, since we control the velocity at the order of magnitude $10^{-3}$, the Reynolds number of each simulation is smaller than 1 (the maximum value is 0.64 ) in this work. This ensures that Darcy's law is valid and the flow is laminar. Therefore, our theory and parameter settings are reasonable and adopted for creeping flow in porous media. When Reynolds number is large, we should consider the BrinkmanStokes flow or turbulent flow. ${ }^{31-33}$ We should also notice that our work may be invalid when Fourier's law breaks down at the nanometer scale. We only discuss the steady results as shown in Figs. 2-6. The proposed theory can also be extended to the unsteady states. The dominant equation is $\rho C \partial T / \partial t+\rho_{f} C_{p, f}(\vec{v} \cdot \nabla T)=\nabla \cdot(\vec{\kappa} \cdot \nabla T)$ where $\rho C=(1-\phi)\left(\rho_{s} C_{s}\right)$ $+\phi\left(\rho_{f} C_{f}\right)$. Here, $\rho_{s}$ and $C_{s}$ are respectively the density and the specific heat of solid material in porous media. Compared with steady states, the density and specific heat should be considered accordingly.

\section{Discussion and conclusion}

In summary, we have proposed a scheme to realize thermal illusion (including transparency, concentrating, and cloaking) for creeping flow in porous media. Such scheme of thermal transparency overcomes the limitations of anisotropy, inhomogeneity, and singularity. This scheme also helps to realize thermal concentrating and cloaking with only constant anisotropic parameters (namely, $\tau_{r r}>\tau_{\theta \theta}$ for concentrating and $\tau_{r r}$ $\ll \tau_{\theta \theta}$ for cloaking). All these three functions do not disturb the temperature, velocity, and heat flux distributions in the background.

Furthermore, we give a new insight on thermal convectiondiffusion process. These two processes are strongly coupled in porous media with creeping flow which presents a huge challenge for controlling the heat flow. However, we decouple thermal conductivity and permeability by considering two independent passive processes in this research, and find that the dominant equations have the same mathematical form. Thus, the effective medium theory can handle both thermal conductivity and permeability, and it is of great significance for practical applications. For example, we can design thermal metamaterials for thermal convection-conduction in porous media just like what we do in thermal conduction to realize desired functions (such as cloaks for hiding objects, concentrators for collecting heat energy, or camouflage for misleading infrared detection). For laboratory experiments, we consider the micro-nano processing technology due to the limitation of system size. We can fabricate porous microfluidic devices by designing patterned porous silicon nitride masters with polydimethylsiloxane(PDMS). Since the designed parameters of the shell are anisotropic for cloak and concentrator, we consider that the shell consists of many micropillars in porous media. PDMS can be made into micropillars and incorporated into porous silicon nitride masters to regulate permeability and thermal conductivity. A recent article gives us a good idea. ${ }^{34}$ We can decompose the shell into many units. Every unit is a micropillar incorporated into the porous media. By designing the permeability and thermal conductivity of every unit and combining all of them, we can realize the desired anisotropy of the shell.

This work provides a direct guidance to experimentally fabricate metamaterials for thermal convection-diffusion, and further works can be expected. For example, it can promote the research on transient thermal convection-diffusion and experimental realizations with the effective medium theory.

\section{Acknowledgments}

F. Y. and L. X. contributed equally to this work. We acknowledge the financial support by the National Natural Science Foundation of China under Grant No. 11725521.

\section{References}

1. H. Bao, J. Chen, X. K. Gu and B. Y. Cao, ES Energy Environ., 2018, 1, 16-55.

2. C. Z. Fan, Y. Gao and J. P. Huang, Appl. Phys. Lett., 2008, 92, 251907.

3. T. Chen, C. N. Weng and J. S. Chen, Appl. Phys. Lett., 2008, 93, 114103.

4. S. Narayana and Y. Sato, Phys. Rev. Lett., 2012, 108, 214303.

5. T. C. Han, T. Yuan, B. W. Li and C. W. Qiu, Sci. Rep., 2013, 3, 1593.

6. H. Y. Xu, X. H. Shi, F. Gao, H. D. Sun and B. L. Zhang, Phys. Rev. Lett., 2014, 112, 054301.

7. T. C. Han, X. Bai, D. L. Gao, J. T. L. Thong, B. W. Li and C. W. Qiu, Phys. Rev. Lett., 2014, 112, 054302.

8. Y. G. Ma, Y. C. Liu, M. Raza, Y. D. Wang and S. L. He, Phys. Rev. Lett., 2014, 113, 205501.

9. Y. Li, X. Y. Shen, Z. H. Wu, J. Y. Huang, Y. X. Chen, Y. S. Ni and J. P. Huang, Phys. Rev. Lett., 2015, 115, 195503.

10. T. C. Han, J. J. Zhao, T. Yuan, D. Y. Lei, B. W. Li and C. W. Qiu, Energ. Environ. Sci., 2013, 6, 35373541

11. M. Moccia, G. Castaldi, S. Savo, Y. Sato and V. Galdi, Phys. Rev. X, 2014, 4, 021025 .

12. T. Y. Chen, C. N. Weng and Y. L. Tsai, J. Appl. Phys., 2015, 117, 054904.

13. X. Y. Shen, Y. Li, C. R. Jiang, Y. S. Ni and J. P. Huang, Appl. Phys. Lett., 2016, 109, 031907.

14. L. J. Xu, S. Yang and J. P. Huang, Phys. Rev. E, 2018, 98, 052128.

15. X. He and L. Z. Wu, Phys. Rev. E, 2013, 88, 033201.

16. L. W. Zeng and R. X. Song, Appl. Phys. Lett., 2014, 104, 201905.

17. L. J. Xu, S. Yang and J. P. Huang, Phys. Rev. Appl., 2019, 11, 034056.

18. T. C. Han, X. Bai, J. T. L. Thong, B. W. Li and C. W. Qiu, Adv. Mater, 2014, 6,1731-1734.

19. Y. Li, X. Bai, T. Z. Yang, H. Luo and C. W. Qiu, Nat. Commun., 2018, 9, 273.

20. R. Hu, S. L. Zhou, Y. Li, D. Y. Lei, X. B. Luo and C. W. Qiu, Adv. Mater, 2018, 30, 1707237.

21. S. L. Zhou, R. Hu and X. B. Luo, Int. J. Heat Mass Transfer, 2018, 127, 607-613.

22. R. Hu and X. B. Luo, Natl. Sci. Rev., 2019, nwz114.

23. L. J. Xu, R. Z. Wang and J. P. Huang, J. Appl. Phys., 2018, 123, 245111.

24. L. J. Xu and J. P. Huang, Phys. Lett. A, 2018, 382, 3313-3316.

25. R. Hu, S. Y. Huang, M. Wang, X. B. Luo, J. Shiomi and C. W. Qiu, $A d v$ Mater, 2019, 31, 1807849.

26. S. Kang, J. Cha, K. Seo, S. Kim, Y. Cha, H. Lee, J. Park and W. Choi, Int. J. Heat Mass Transfer, 2019, 130, 469-482.

27. G. L. Dai, J. Shang and J. P. Huang, Phys. Rev. E, 2018, 97, 022129.

28. G. L. Dai and J. P. Huang, J. Appl. Phys., 2018, 124, 235103.

29. L. J. Xu, S. Yang and J. P. Huang, Phys. Rev. Appl., 2019, 11, 054071.

30. http://www.comsol.com/.

31. Y. A. Urzhumov and D. R. Smith, Phys. Rev. E, 2012, 86, 056313.

32. P. T. Bowen, Y. A. Urzhumov and D. R. Smith, Phys. Rev. E, 2015, 92, 063030 .

33. D. R. Culver, E. Dowell, D. R. Smith, Y. A. Urzhumov and A. Varghese, J Fluids, 2016, 2016, 1-15

34. J. Park, H. R. Youn and Y. S. Song, Phys. Rev. Lett., 2019, 123, 074502.

Publisher's Note Engineered Science Publisher remains neutral with regard to jurisdictional claims in published maps and institutional affiliations. 